\title{
Contrast-enhanced Ultrasound Imaging Using Capacitive Micromachined Ultrasonic Transducers
}

Sigrid Husebø Øygard ( $\nabla$ shoy@dtu.dk)

Technical University of Denmark

Martin L. Ommen

Technical University of Denmark

Borislav G. Tomov

Technical University of Denmark

Søren E. Diederichsen

Technical University of Denmark

Erik V. Thomsen

Technical University of Denmark

Niels B. Larsen

Technical University of Denmark

Matthias B. Stuart

Technical University of Denmark

Jørgen A. Jensen

Technical University of Denmark

\section{Research Article}

Keywords: Contrast-enhanced ultrasound imaging, capacitive micromachined ultrasonic transducers, amplitude modulation

Posted Date: November 22nd, 2021

DOI: https://doi.org/10.21203/rs.3.rs-1085754/v1

License: (c) (i) This work is licensed under a Creative Commons Attribution 4.0 International License.

Read Full License 


\section{RESEARCH}

2

3

Contrast-enhanced ultrasound imaging using

5 ${ }_{7}^{6}$ Capacitive micromachined ultrasonic transducers

8Sigrid H. Øygard ${ }^{1 *}$, Martin L. Ommen ${ }^{3}$, Borislav G. Tomov ${ }^{1}$, Søren E. Diederichsen ${ }^{3}$, Erik V. Thomsen ${ }^{3}, 8$ ${ }_{9}$ Niels B. Larsen ${ }^{2}$, Matthias B. Stuart ${ }^{1}$ and Jørgen A. Jensen ${ }^{1}$

10

Abstract

Capacitive micromachined ultrasonic transducers (CMUTs) have a nonlinear relationship between the applied voltage and the emitted signal, which is detrimental to conventional contrast enhanced ultrasound (CEUS) techniques. Instead, a three-pulse amplitude modulation (AM) sequence has been proposed, which is not adversely affected by the emitted harmonics. In this paper, this is shown theoretically, and the performance of the sequence is verified using a $4.8 \mathrm{MHz}$ linear CMUT array, and a comparable lead zirconate titanate (PZT) array, across $6 \mathrm{~V}$ to $60 \mathrm{~V}$ applied $\mathrm{AC}$ voltage. CEUS images of the contrast agent SonoVue flowing through a 3D printed hydrogel phantom showed an average enhancement in contrast-to-tissue ratio (CTR) between B-mode and CEUS images of $49.9 \mathrm{~dB}$ and $37.4 \mathrm{~dB}$ for the PZT array and CMUT, respectively. Furthermore, hydrophone recordings of the emitted signals showed that the nonlinear emissions from the CMUT did not significantly degrade the cancellation in the compounded AM signal, leaving an average of $2 \%$ of the emitted power between $26 \mathrm{~V}$ and $60 \mathrm{~V} \mathrm{AC}$. Thus, it is demonstrated that CMUTs are capable of CEUS imaging independent of the applied excitation voltage when using a three-pulse AM sequence. 
${ }^{1}$ Keywords: Contrast-enhanced ultrasound imaging, ${ }^{2}$ capacitive micromachined ultrasonic transducers,

3 amplitude modulation

4

5

\section{Introduction}

${ }_{7}$ Recently, capacitive micromachined ultrasonic trans${ }_{8}$ ducers (CMUTs) have become available as an alterna${ }_{9}$ tive to the more conventional piezoelectric transducers ${ }_{10}[1,2]$. CMUTs offer sensitivity features that are partic${ }_{11}$ ularly suitable for microbubble contrast-enhancement ${ }_{12}$ imaging, with low transmit pressure, yet high receive ${ }_{13}$ sensitivity [1]. Contrast enhanced ultrasound (CEUS) ${ }_{14}$ imaging with microbubble contrast agents is used clin${ }_{15}$ ically to study the blood's wash-in and wash-out time ${ }_{16}$ in organs [3], to quantify the blood-flow velocity using ${ }_{17}$ time-intensity curves [4], and, most recently, to im${ }_{18}$ age blood-flow through capillaries beyond the diffrac${ }_{19}$ tion limit, through super-resolution ultrasound imag${ }_{20}$ ing (SRI) $[5,6]$. CEUS imaging with a CMUT is a ${ }_{21}$ combination of two nonlinear processes; the spectral ${ }_{22}$ content of the emission from a CMUT is nonlinearly ${ }_{23}$ related to its excitation voltage [7], and the spectral ${ }_{24}$ content of the scattering of the excitation by the mi${ }_{25}$ crobubble contrast agents is nonlinearly related to the ${ }_{26}$ acoustic pressure [8]. This combination poses some ${ }_{27}$ challenges to the conventional CEUS techniques, and ${ }_{28}$ the viability of CEUS imaging using CMUTs has been ${ }_{29}$ questioned [9].

${ }_{30}$ CMUTs emit ultrasound by applying an AC volt${ }_{31}$ age potential across thin plates suspended on top of ${ }_{32}$ nanometer to micrometer sized cavities. The technol${ }_{33}$ ogy offers great design flexibility [10], making it possi${ }_{34}$ ble to produce transducers with several advantageous ${ }_{35}$ features for CEUS imaging. Firstly, the transducer can ${ }_{36}$ achieve wide bandwidth, resulting in high axial resolu$37^{*}$ Correspondence: shoy@dtu.dk

${ }^{1}$ Center for Fast Ultrasound Imaging, Department of Health Technology,

Technical University of Denmark, Kgs. Lyngby, Denmark

${ }^{39}$ Full list of author information is available at the end of the article tion [10]. For SRI, where separation of the microbub- ${ }^{1}$ bles within each image frame is essential, this could en- ${ }^{2}$ able SRI with a higher number of resolved microbub- ${ }^{3}$ bles within each frame, and thus a lower acquisition ${ }^{4}$ time. The wide bandwidth would also facilitate detec- ${ }^{5}$ tion of higher harmonics scattered by the microbub- ${ }^{6}$ bles, often utilized in the pulse inversion (PI) tech- ${ }^{7}$ nique. Secondly, CMUTs can produce a low emitted ${ }^{8}$ pressure, suitable for exciting microbubbles without ${ }^{9}$ destroying them [11], whilst maintaining a high re- ${ }^{10}$ ceive sensitivity [12]. However, the drawback of $\mathrm{CMUT}^{11}$ technology is that the electrostatic force driving the ${ }^{12}$ excitation causes the emitted signal to contain con- ${ }^{13}$ siderable harmonic components [13]. The amount of ${ }^{14}$ harmonic content produced by the transducer is de- ${ }^{15}$ pendent on the ratio of the applied $\mathrm{AC}$ voltage to the ${ }^{16}$ applied DC voltage [7], which renders the transducer ${ }^{17}$ incapable of removing of tissue-generated signals using ${ }^{18}$ conventional CEUS techniques.

CEUS imaging achieves high contrast by removing ${ }^{21}$ the signal scattered by the tissue from that scattered ${ }^{22}$ by the microbubbles. The most common techniques ${ }^{23}$ used to remove the tissue signal are PI, amplitude ${ }^{24}$ modulation (AM), and singular value decomposition ${ }^{25}$ (SVD). The first two utilize the fact that when $a^{26}$ bubble is insonified, it will scatter the sound nonlin- ${ }^{27}$ early [14]. SVD is a spatiotemporal filtering technique, ${ }^{28}$ which utilizes the bubble's high scattered intensity and ${ }^{29}$ temporally transient behavior to separate the bubbles ${ }^{30}$ from the tissue [5]. These techniques are thus capable ${ }^{31}$ of removing the linearly scattered signals caused by the ${ }^{32}$ tissue surrounding the contrast agents. PI combines ${ }^{33}$ the responses from two excitations with opposite signs ${ }^{34}$ [15]. Conventionally, AM is achieved by combining the ${ }^{35}$ response from two excitations, one with half the ampli- ${ }^{36}$ tude of the next [16]. Both the difference in amplitude ${ }^{37}$ and difference in polarity will cause the CMUT to emit ${ }^{38}$ harmonics that differ between the emissions, which ${ }^{39}$ 
${ }^{1}$ conventional AM and PI will not be able to remove.

${ }^{2}$ Using such sequences with CMUTs will therefore re-

${ }^{3}$ sult in poor contrast in the CEUS images $[17,18]$. Sev-

${ }^{4}$ eral attempts have been made to overcome this issue

${ }^{5}$ by altering the frequency content of the emitted pulse

${ }^{6}[19,20]$, but such approaches are not necessarily com${ }^{7}$ patible with the bipolar pulsers often found in com-

${ }^{8}$ mercial scanners. Moreover, these methods rely on all

${ }^{9}$ elements in the probe behaving identically, which is

${ }^{10}$ often not the case. However, Fouan and Bouakaz [18]

${ }^{11}$ have proposed that CEUS imaging can be obtained

${ }^{12}$ using simple sinusoidal excitations by implementing a

${ }^{13}$ three-pulse AM sequence. In this sequence, the differ-

${ }^{14}$ ence in amplitude between emissions, which is needed

${ }^{15}$ to preserve the nonlinear back-scattering from the mi-

${ }^{16}$ crobubbles, is achieved by emitting once with the full

${ }^{17}$ active aperture, once with the odd-numbered elements,

${ }^{18}$ and once with the even-numbered elements. Fouan and

${ }^{19}$ Bouakaz [18] shows that for a single transmit voltage

${ }^{20}$ of $20 \mathrm{~V}$ applied to a CMUT, a $15 \mathrm{~dB}$ improvement in

${ }^{21}$ contrast-to-tissue ratio (CTR) was achieved, compared

${ }^{22}$ with an AM sequence with scaled voltage. A similar

${ }^{23}$ approach has been studied in [17], where it was shown

${ }^{24}$ that a two-pulse AM sequence with halving of the ac-

${ }^{25}$ tive elements resulted in better contrast than conven-

${ }^{26}$ tional PI imaging. The contrast of CEUS images is

${ }^{27}$ influenced by the power of the AM signal compared

${ }^{28}$ with the power of any remaining signal scattered by

${ }^{29}$ tissue, often quantified by CTR [21]. Since the applied

${ }^{30}$ voltage and polarity to each emitting element is con-

${ }^{31}$ stant for all emissions in these modified AM sequences,

${ }^{32}$ the theory predicts that the emitted signals therefore

${ }^{33}$ contain the same harmonics. This indicates that the

${ }^{34}$ increasing harmonic content resulting from increased

${ }^{35}$ applied AC voltage does not affect the performance of

${ }^{36}$ the imaging technique.

37

38 This paper investigates the two-pulse and three-

${ }^{39}$ pulse AM sequence at a wide range of transmit volt- ages, corresponding to varying nonlinear components ${ }^{1}$ in the emissions. It is hypothesized that the amount ${ }^{2}$ of nonlinear harmonics in the emitted signal does not ${ }^{3}$ affect the efficiency of these modified AM techniques. ${ }^{4}$ This is demonstrated theoretically, and verified with ${ }^{5}$ measurements. The method's ability to cancel linear ${ }^{6}$ components is measured using hydrophone recordings, ${ }^{7}$ and its contrast enhancement is assessed using $\mathrm{CEUS}^{8}$ imaging of a microflow phantom with SonoVue con- ${ }^{9}$ trast agents. The measurements are made using a lin- ${ }^{10}$ ear CMUT array produced in house, and, for refer- ${ }^{11}$ ence, using a corresponding commercial lead zirconate ${ }^{12}$ titanate (PZT) array.

\section{Theory 20}

\subsection{Emission from a CMUT 22}

The propagation of sound waves from a CMUT ar- ${ }^{24}$ ray is induced by applying an $\mathrm{AC}$ voltage, $V_{A C}$, across ${ }^{25}$ the CMUT cells. This causes a time-varying deflection, ${ }^{26}$ driven by electrostatic force on the cell plates. [22]. The ${ }^{27}$ acoustic pressure radiated from a circular cell plate is ${ }^{28}$ proportional to the resulting displacement of the plate ${ }^{29}$ [23], and the relationship between the applied volt- ${ }^{30}$ age and the displacement is governed by a nonlinear ${ }^{31}$ differential equation [24]. Analytic evaluation of this ${ }^{32}$ equation is not straight forward, but several authors ${ }^{33}$ have studied the relationship numerically $[22,24,25] .^{34}$ It has been shown that the emitted sound pressure ${ }^{35}$ from CMUTs contain harmonics to the applied exci-36 tation frequency $[24,26]$. Thus, when the applied $V_{A C}{ }^{37}$ is a simple sinusoid at some excitation frequency, $\omega_{0}{ }^{38}$ the resulting emitted pressure, $p(t)$, can be expressed ${ }^{39}$ 


$$
\begin{aligned}
& { }^{1} \text { as } \\
& 2 \\
& { }^{3} p(t)=\alpha_{0} \sin \left(\omega_{0} t\right) \\
& 4 \\
& 5 \\
& 6 \\
& 7 \\
& +\sum_{h=2}^{\infty} \alpha_{h} \sin \left(h \omega_{0} t+\phi_{h}\right),\left\{\begin{array}{l}
h \in \mathbb{Z}, \\
\alpha_{h} \in \mathbb{R}
\end{array}\right. \\
& { }_{9}^{8}=p_{L}(t)+\sum_{h=2}^{\infty} p_{H}(t)
\end{aligned}
$$

10

${ }^{11}$ Here, $t$ is time, $\alpha$ is some amplitude, $h$ is a harmonic 12index, $\phi$ is a phase difference. Since $\alpha_{h}$ can be any ${ }^{13}$ real number, including zero, this notation is applicable ${ }^{14}$ even when only certain harmonics are present in the ${ }^{15}$ emitted signal. A compact form of (1) is given in (2), ${ }^{16}$ where $p_{L}(t)$ is the linear part, i.e. the component of 17the signal which has the same frequency content as 18the applied $\omega_{0}$, and $\sum p_{H}(t)$ is the harmonics. The ${ }^{19}$ amplitude and phase of the harmonics are determined 20 by the complex dynamics of the CMUT array, and are ${ }^{21}$ not further described here. Notably, it has been shown 22that the amount of harmonic content in the emitted ${ }^{23}$ pressure wave depends on the ratio of the amplitude ${ }^{24}$ of the applied $V_{A C}$ to the applied DC voltage [26], and ${ }^{25} \mathrm{CMUTs}$ are therefore said to be inherently nonlinear.

${ }^{26}$ However, it will here be shown that the nature of the ${ }^{27}$ harmonics does not affect the effectiveness of the three${ }^{28}$ pulse AM sequence.

29 In the three-pulse AM sequence proposed by Fouan and Bouakaz [18], a pulse of full amplitude, $p_{A}$, is 31 emitted, followed by two pulses of halved amplitude, ${ }^{32} p_{B}$ and $p_{C}$. The applied voltage is constant across all ${ }^{33}$ three emissions, and the two half amplitudes are made ${ }^{34}$ by halving the number of active elements compared 35 with the full emission, spread across the same active 36 aperture width. Thus, the three emitted pulses are 37

38 39

$$
p_{A}(t)=\alpha_{0} \sin \left(\omega_{0} t\right)+\sum_{h=2}^{\infty} \alpha_{h} \sin \left(h \omega_{0} t+\phi_{h}\right),
$$

$$
\begin{aligned}
p_{B}(t)=0.5\left[\alpha_{0} \sin \left(\omega_{0} t\right)\right. & 1 \\
& \left.+\sum_{h=2}^{\infty} \alpha_{h} \sin \left(h \omega_{0} t+\phi_{h}\right)\right], \\
p_{C}(t)=0.5\left[\alpha_{0} \sin \left(\omega_{0} t\right)\right. & 3 \\
& \left.+\sum_{h=2}^{\infty} \alpha_{h} \sin \left(h \omega_{0} t+\phi_{h}\right)\right] .
\end{aligned}
$$

\subsection{The amplitude modulation technique}

8

When the microbubbles are insonified with an ultra- ${ }_{10}$ sound pulse, the reflections contain harmonics of the ${ }_{11}$ incident frequency. Eckersley et al. [11] show that the ${ }_{12}$ nonlinear reflections can be modeled as a polynomial ${ }_{13}$ expansion of the incident wave, as

$y(t)=a_{1} p_{i}(t)+a_{2}{p_{i}}^{2}(t)+a_{3} p_{i}{ }^{3}(t)+\ldots+a_{\infty} p_{i}{ }^{\infty}(t) .(6)_{16}^{15}$

Here, $y(t)$ is the reflected signal, $p_{i}(t)$ is the incident ${ }^{17}$ pressure wave on the bubble, and $a_{i}$ are reflection co- ${ }^{18}$ efficients determining the different nonlinear contri- ${ }^{19}$ butions, where $a_{1}$ represents the linear reflection and ${ }^{20}$ $a_{i>1}$ represents the nonlinear reflections. To suppress ${ }^{21}$ the linear component of the emitted signal, while pre- ${ }^{22}$ serving the nonlinear components at the fundamental, ${ }^{23}$ the reflected signals are combined as

$$
y_{A M}(t)=y_{A}(t)-y_{B}(t)-y_{C}(t)
$$
where $y_{A M}$ is the remaining three-pulse amplitude ${ }_{28}$ modulation signal, and $y_{A}, y_{B}, y_{C}$ are the scattered 29 signals caused by the three emissions. If instead, only ${ }_{30}$ 2 emissions are used the combination is made as 31

$$
y_{A M}(t)=y_{A}(t)-2 \cdot y_{B}(t) . \quad(8)_{33}^{32}
$$

\subsection{Amplitude modulation with a CMUT 34} By assuming that no distortion of the signal occurs ${ }^{35}$ between the emission and the bubble position, we can ${ }^{36}$ apply (6) to the tree emissions given in (3) - (5) before $^{37}$ applying (7). By expressing the pressure waves in their ${ }^{38}$ compact form and limiting the polynomial expansion ${ }^{39}$ 
${ }^{1}$ to third order for simplicity, this gives

2

$3 y_{A M}(t)=a_{1} p_{A}+a_{2} p_{A}^{2}+a_{3} p_{A}^{3}-a_{1} p_{B}-a_{2} p_{B}^{2}$

$4-a_{3} p_{B}{ }^{3}-a_{1} p_{C}-a_{2} p_{C}{ }^{2}-a_{3} p_{C}{ }^{3}$.

5

Then, by expanding to the long form of (3) - (5), set-

ting $\alpha_{0}=1$, working through the algebra (shown in

${ }_{8}$ more detail in the appendix) gives

${ }^{9} y_{A M}(t)=0.5 a_{2} \sin \left(\omega_{0} t\right)^{2}$

$$
\begin{array}{ll}
10 & +0.5 a_{2}\left(\sum_{h=2}^{\infty} \alpha_{h} \sin \left(h \omega_{0} t+\phi_{h}\right)\right)^{2} \\
12 & +a_{2} \sin \left(\omega_{0} t\right)\left(\sum_{h=2}^{\infty} \alpha_{h} \sin \left(h \omega_{0} t+\phi_{h}\right)\right) \\
13 & +2.25 a_{3} \sin \left(\omega_{0} t\right)\left(\sum_{h=2}^{\infty} \alpha_{h} \sin \left(h \omega_{0} t+\phi_{h}\right)\right)^{2} \\
15 & +2.25 a_{3} \sin \left(\omega_{0} t\right)^{2}\left(\sum_{h=2}^{\infty} \alpha_{h} \sin \left(h \omega_{0} t+\phi_{h}\right)\right)^{3} \\
17 & +0.75 a_{3}\left(\sum_{h=2}^{\infty} \alpha_{h} \sin \left(h \omega_{0} t+\phi_{h}\right)\right)^{3} \\
19 & +0.1875 a_{3} \sin \left(3 \omega_{0} t\right) \\
21 & +1.5 a_{3} \sin \left(\omega_{0} t\right) . \\
22 &
\end{array}
$$$$
19
$$$$
23
$$

${ }^{24}$ Two important observations can be made about (10). ${ }^{25}$ Firstly, it reveals a subtle, but, essential property of ${ }^{26} \mathrm{AM}$ imaging; the remaining signal lacks any linearly ${ }^{27}$ scattered components, yet, the last term of (10) con${ }^{28}$ tains energy at the fundamental frequency. The re${ }^{29}$ flection coefficient $a_{3}$ indicates that this component ${ }^{30}$ is caused by the nonlinear scattering properties of the ${ }^{31}$ microbubbles. Secondly, it is observed that this can${ }^{32}$ cellation of the linearly scattered signal was attained ${ }^{33}$ despite the nonlinear nature of the CMUT. Although ${ }^{34}$ harmonics to the fundamental frequency are emitted, ${ }^{35}$ as seen in (3) - (5), (10) does not contain any emit${ }^{36}$ ted harmonics with the linear reflection coefficient $a_{1}$. ${ }^{37}$ This shows that when the emitted signals are reflected ${ }^{38}$ only linearly, the AM compounding cancels all of these ${ }^{39}$ terms. Some emitted harmonics remain in the $y_{A M}$ sig- nal, but, these terms all carry the reflection coefficients ${ }^{1}$ $a_{2}$ or $a_{3}$, meaning that the signals have been nonlin- ${ }^{2}$ early scattered by the microbubble. Notably, the fact ${ }^{3}$ that these emitted harmonics remain in the $y_{A M}$ sig- $^{4}$ nal has not impaired the efficiency of the AM theory. ${ }^{5}$ Moreover, although the amplitude and phase of the ${ }^{6}$ emitted harmonics will be affected by the amplitude ${ }^{7}$ of $V_{A C}$, this does not affect the cancellation of linearly ${ }^{8}$ scattered signals. Because of this, when using the $\mathrm{AM}^{9}$ modality to image in vivo with a CMUT, it is possible ${ }^{10}$ to filter out linear scattering from tissue whilst attain- ${ }^{11}$ ing reflections from the microbubbles at the emitted ${ }^{12}$ frequency. Thus, it is shown theoretically that CEUS ${ }^{13}$ imaging with a CMUT using the three-pulse AM se- ${ }^{14}$ quence is achievable. 15

\section{Methods 17} To verify the outcome of the theoretical evaluation ${ }^{18}$ shown in Section 2, the ability of a linear CMUT array ${ }^{19}$ to cancel received linear components while preserving ${ }^{20}$ nonlinearly scattered signal was assessed using three ${ }^{21}$ measurement set-ups. Firstly, an experimental set-up ${ }^{22}$ was designed to quantify CTR. Section 3.1 outlines $^{23}$ the design of the set-up. Contrast enhanced AM im- ${ }^{24}$ ages were collected using a synthetic aperture imag- ${ }^{25}$ ing sequence on a CMUT and a PZT probe, further ${ }^{26}$ described in Section 3.2. Subsequently, the CTR was ${ }^{27}$ calculated for all the collected images, as detailed in $^{28}$ Section 3.3. Secondly, Section 3.4 describes how the ${ }^{29}$ emitted signal from the transducers were recorded us- ${ }^{30}$ ing a hydrophone, and used to determine the peak- ${ }^{31}$ negative-pressure (PNP) and the power annihilation ${ }^{32}$ ratio. Lastly, Section 3.5 describes how the signal-to- ${ }^{33}$ noise ratio of the two probes were evaluated to fairly ${ }^{34}$ compare the CMUT's and PZT arrays's performance. ${ }^{35}$

\subsection{CEUS imaging set-up 37}

The experimental set-up used to quantify CTR con- ${ }^{38}$ sisted of a microflow phantom and a block of tissue- ${ }^{39}$ 


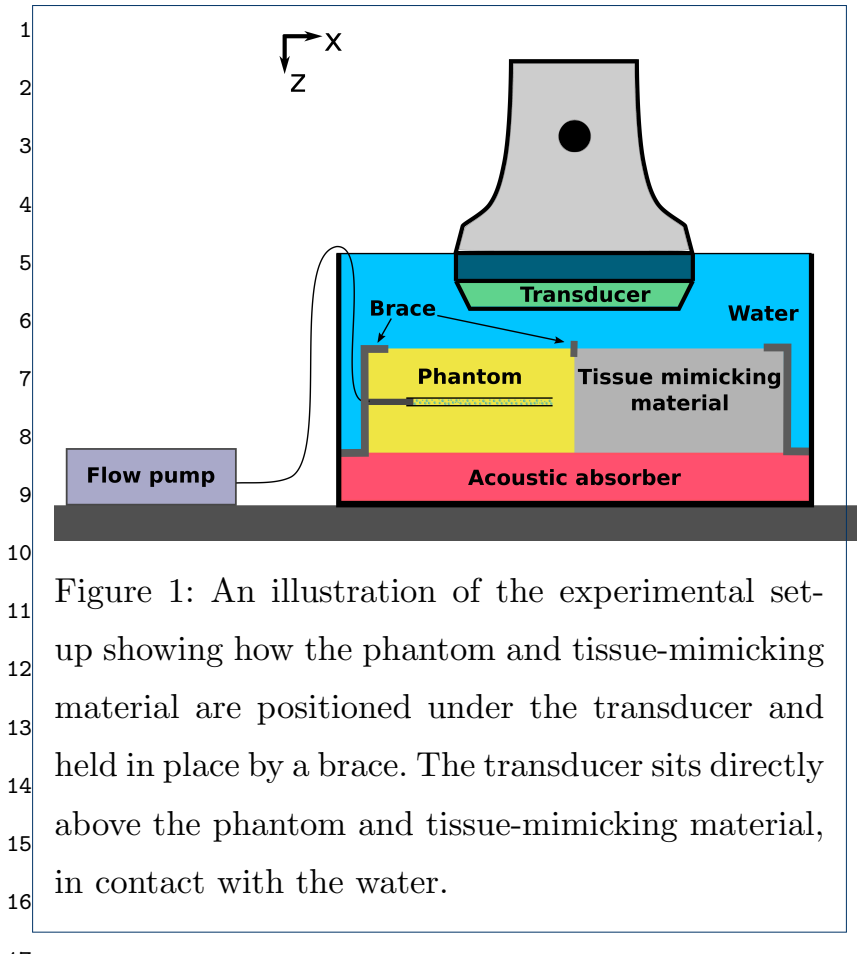

17

18

19

20

${ }^{21}$ mimicking material. An illustration of the measure${ }^{22}$ ment set-up can be seen in Fig. 1. The microflow phan${ }^{23}$ tom was $3 \mathrm{D}$ printed in a PEGDA $700 \mathrm{~g} \mathrm{~mol}^{-1}$ hydro${ }^{24}$ gel block using stereo-lithography. A hollow channel of ${ }^{25} 200 \mathrm{\mu m}$ in diameter was printed inside the phantom, as ${ }^{26}$ shown in Fig. 2. The fabrication process of the phan${ }^{27}$ tom has been described in [27]. The phantom was held ${ }^{28}$ in place by a brace next to a block of tissue-mimicking ${ }^{29}$ material made of polyvinyl alcohol cryogel [28, 29], ${ }^{30}$ resulting in scattering properties similar to that of hu${ }^{31} \mathrm{man}$ tissue. The ensemble was placed in a water bath, ${ }^{32}$ which was lined with an acoustic absorber. The phan${ }^{33}$ tom was positioned so that the center of the flow chan${ }^{34} \mathrm{nel}$ was $25 \mathrm{~mm}$ below the surface of the transducer. A ${ }^{35}$ low pressure flow pump (neMESYS 290N, Cetoni) fit${ }^{36}$ ted with a syringe of SonoVue microbubble contrast ${ }^{37}$ agents, diluted 1:10 with saline, was connected to the ${ }^{38}$ phantom and used to pump microbubbles through the ${ }^{39}$ phantom.
Table 1: Specifications of the transducers.

\begin{tabular}{|l|l|l|}
\hline & CMUT & PZT \\
\hline Total number of elements & 192 & 192 \\
\hline Center frequency & $4.8 \mathrm{MHz}$ & $5.2 \mathrm{MHz}$ \\
\hline Pitch $(p)$ & $0.2 \mathrm{~mm}$ & $0.2 \mathrm{~mm}$ \\
\hline Kerf & $0.02 \mathrm{~mm}$ & $0.02 \mathrm{~mm}$ \\
\hline Maximum tolerable AC voltage & $60 \mathrm{~V}$ & $100 \mathrm{~V}$ \\
\hline Applied DC voltage & $190 \mathrm{~V}$ & $\mathrm{~N} / \mathrm{A}$ \\
\hline
\end{tabular}

\subsection{Image acquisition}

The phantom and tissue-mimicking material was im-12 aged using a linear CMUT [30] and, as a reference, 13 a commercial linear PZT array of equivalent design ${ }_{14}$ dimensions, using a Verasonics research scanner (Ve-15 rasonics Vantage 256). The specifications of the probes 16 are given in Table 1. The set-up was imaged using $\mathrm{a}_{17}$ synthetic aperture sequence [31] with low intensity di-18 verging wave propagation. A summary of the sequence 19 parameters are given in Table 2. The transducers emit-20 ted a 2 cycle sine wave at $4.8 \mathrm{MHz}$, with 32 active el- 21 ements in each full amplitude emission, and with $\mathrm{a}_{22}$ pulse repetition frequency of $1000 \mathrm{~Hz}$. The sequence 23

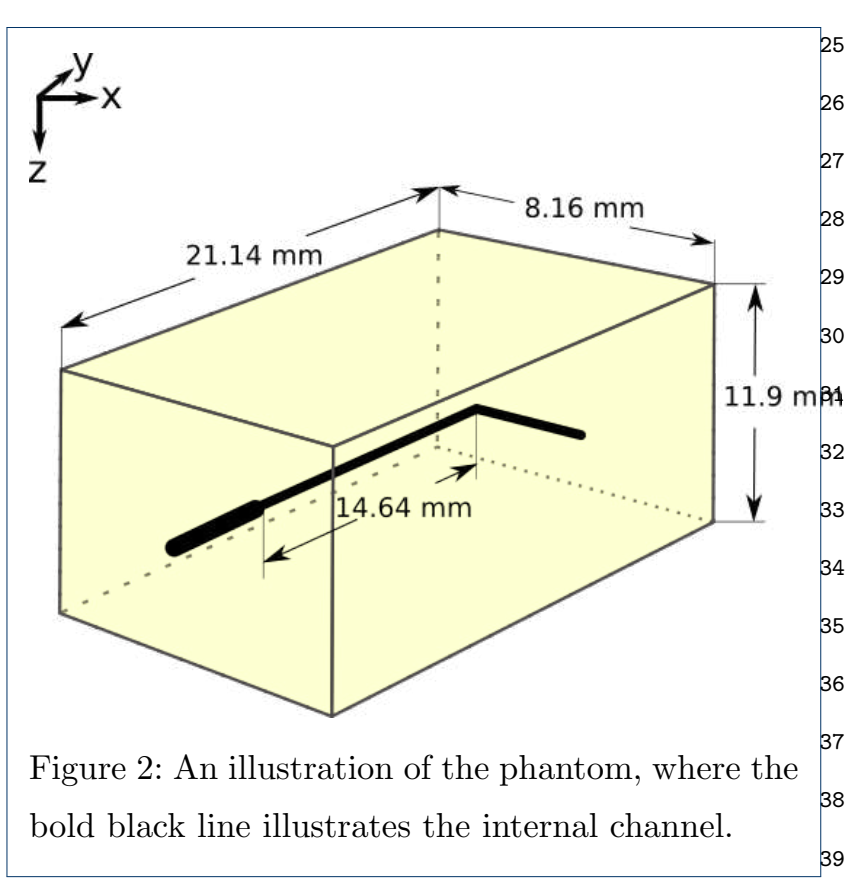


${ }^{1}$ Table 2: Parameters of the synthetic aperture se2 quence.

\begin{tabular}{|c|c|c|}
\hline 3 & Pulse repetition frequency & $1000 \mathrm{~Hz}$ \\
\hline 4 & Number of active elements & 32 \\
\hline 5 & Emission frequency & $4.8 \mathrm{MHz}$ \\
\hline 6 & Focal distance & $-16 \mathrm{~mm}$ \\
\hline 7 & Receive F-number & 1 \\
\hline 8 & Number of emissions $(n)$ & 13 \\
\hline 9 & Number of cycles in emission & 2 \\
\hline 10 & $\begin{array}{l}\text { Number of elements } \\
\text { separating emissions }(s)\end{array}$ & 13 \\
\hline
\end{tabular}

12

13 consisted of emissions from $n=13$ virtual sources ${ }^{14}$ placed $16 \mathrm{~mm}$ behind the transducer surface. The lat${ }^{15}$ eral positions of the virtual sources were given by 16$$
17 \quad x_{i}=s \cdot i \cdot p, \forall i \in \mathbb{Z}: i \in\left[-\frac{(n-1)}{2}, \frac{(n-1)}{2}\right],
$$$$
18
$$

19

${ }^{20}$ where $s$ denotes the number of elements separating ${ }^{21}$ the lateral positions of two virtual sources, $i$ is emis${ }^{22}$ sion index, $p$ is the transducer pitch, and $x_{i}=0$ lays ${ }^{23}$ in the middle of the transducer. The three emissions ${ }^{24}$ described in $(3)$ - (5) are referred to as the minor se${ }^{25}$ quence. The minor sequence was emitted once by each ${ }^{26}$ virtual source at each $x_{i}$ to make a full image frame.

27 The sequence was used to quantify how the CTR in ${ }^{28}$ the resulting images vary with the applied AC voltage. ${ }^{29}$ The lowest $V_{A C}$ was $6 \mathrm{~V}$, at which the imaging system ${ }^{30}$ approaches its noise floor. For the CMUT, $V_{A C}$ was ${ }^{31}$ then increased in steps of $10 \mathrm{~V}$ until the maximum tol${ }^{32}$ erable AC voltage of the probe, as given in Table 1, ${ }^{33}$ was reached. Additionally, a constant DC voltage of ${ }^{34} 190 \mathrm{~V}$ was applied to the CMUT, as specified in [30]. ${ }^{35}$ For the PZT array, the same voltage range was used, ${ }^{36}$ from $6 \mathrm{~V}$ to the probe's maximum tolerable voltage. ${ }^{37}$ But, the step size was reduced to $3 \mathrm{~V}$ between $6 \mathrm{~V}$ and ${ }^{38} 15 \mathrm{~V}$. This was done to ensure a good population of ${ }^{39}$ data-points within the overlapping range of PNP for the two probes, since the relationship between applied ${ }^{1}$ voltage and PNP is not linear. 2

The SonoVue solution was allowed to flow continuously through the phantom channel at $0.6 \mathrm{\mu Ls}^{-1}$, while 10 image frames were collected at each $V_{A C}$. To avoid degradation of the microbubbles during the col- ${ }^{6}$ lection of the 10 frames, a pause of $15 \mathrm{~s}$ was inserted ${ }^{7}$ between each frame to allow a fresh batch of microbubbles to flow into the phantom. A fresh syringe of diluted contrast agents were prepared directly prior to each data-set acquisition. Due to the higher number of ${ }^{11}$ AC voltages, the acquisition using the PZT probe was ${ }^{12}$ divided into two data-sets to make the time each syringe spent in the infusion pump comparable for each ${ }^{14}$ data-set. Thus, 3 data-sets were acquired; $6 \mathrm{~V}$ to $60 \mathrm{~V}^{15}$ with the CMUT, and $6 \mathrm{~V}$ to $26 \mathrm{~V}$ as well as $26 \mathrm{~V}$ to ${ }^{16}$ $90 \mathrm{~V}$ with the PZT array.

\subsection{Calculation of CTR}

The collected data from both probes were matched ${ }^{21}$ filtered and beamformed using delay-and-sum beamforming. CEUS images were composed using both $(7)^{23}$ and (8). The two-pulse AM sequence, which was also ${ }^{24}$ studied by the authors in [32], is included for comparison with the three-pulse AM proposed by Fouan and ${ }^{26}$ Bouakaz [18], to study how an additional emission in ${ }^{27}$ the minor sequence affects the image quality. In ad- ${ }^{28}$ dition, the data from every full amplitude emission in ${ }^{29}$ the minor sequence was used to compose B-mode images, for comparison. For each composed image, the ${ }^{31}$ CTR was calculated as

$$
C T R=\frac{P_{B}}{P_{T M M}}
$$

where $P_{B}$ is the average power of the signal scattered ${ }^{37}$ by the bubbles and $P_{T M M}$ is the average power scat-38 tered by the tissue-mimicking material. The average ${ }^{39}$ 
${ }^{1}$ power was calculated as

2

$3 \quad P=\frac{1}{L_{2}-L_{1}} \frac{1}{k_{2}-k_{1}} \sum_{l=L_{1}}^{L_{2}} \sum_{m=k_{1}}^{k_{2}} y_{l, m}^{2}$,

5

${ }_{6}$ where $y$ is the signal in a single beamformed line of ${ }_{7}$ data, $m$ is the sample number, $k_{1}$ and $k_{2}$ are sam${ }_{8}$ ple numbers determining the axial position for the 9signal, and $L_{1}$ and $L_{2}$ are line numbers. Thus, the ${ }_{10}$ power was calculated in two rectangular regions-of${ }_{11}$ interest (ROIs). After beamforming the collected data, ${ }_{12}$ the ROIs used to calculate power in (13) were drawn ${ }_{13}$ around the channel in the phantom. The ROI in the ${ }_{14}$ tissue-mimicking phantom was taken to be the same ${ }_{15}$ size as the channel ROI, and placed at the same depth, ${ }_{16}$ in the middle of the tissue-mimicking phantom.

17

\subsection{Hydrophone recordings}

${ }^{19}$ The derivation of (10) assumes that the same signal ${ }^{20}$ is produced by the full emission as the two half emis${ }^{21}$ sions combined. Array effects could arguably make the ${ }^{22}$ assumption fail if emission from one CMUT cell af${ }^{23}$ fects the emission from a neighbouring cell. It has been ${ }^{24}$ shown that the emission from an array of CMUTs ${ }^{25}$ is dependent on the distance between adjacent cells ${ }^{26}[25]$. This distance varies between the emissions in ${ }^{27}$ the proposed sequences; in the full amplitude emis${ }^{28}$ sions the distance is one pitch, while in the half am${ }^{29}$ plitude emissions it is 2 . pitch. To evaluate if this ${ }^{30}$ effect adversely affects the similarity of the emitted ${ }^{31}$ pulses, and thus the cancellation of received linear ${ }^{32}$ terms, the signals emitted by each virtual source were ${ }^{33}$ recorded using a hydrophone. The hydrophone was ${ }^{34}$ placed in a water tank directly beneath the transducer, ${ }^{35}$ at $(y, z)=(0,25) \mathrm{mm}$ and moved to the $x$-positions ${ }^{36}$ corresponding to each virtual source, given by (11). ${ }^{37}$ The hydrophone recorded the minor sequence using ${ }^{38}$ all the applied AC voltages, from each virtual source. ${ }^{39}$ Then, the sequence's ability to cancel linear terms was quantified by defining an annihilated power ratio as ${ }^{1}$

$$
\text { Annihilated power ratio }=\frac{\Sigma p_{A}^{2}-\Sigma p_{A M}^{2}}{\Sigma p_{A}^{2}}
$$

Here, the numerator contains the remaining AM sig- ${ }_{6}^{5}$ nal subtracted from the full emitted signal and thus gives the power of the annihilated signal. For each $x^{-}$ position and applied $V_{A C}$, the recordings of the minor sequence were used to compose the compounded ${ }^{9}$ emitted AM signal, $p_{A M}$, according to (7), and the annihilated power ratio. This evaluation will be affected ${ }^{11}$ by nonlinear propagation in the water. The evaluation was therefore performed using both the PZT array and ${ }^{13}$ CMUT for comparison, to distinguish effects caused by CMU for comparison, to distinguish effects caused by nonlinear propagation from the effects caused by nonlinear excitation.

In addition, the measured hydrophone signals were ${ }_{18}$ used to quantify the PNP caused by each applied $V_{A C_{19}}$ for both transducers. The minimum of the recording of the full emission was found from the recording beneath ${ }_{21}$ each virtual source and averaged to give the PNP.

\subsection{Quantifying signal-to-noise ratio}

Because the imaging performance of a CMUT and $\mathrm{a}^{25}$ PZT transducer are to be compared, it is prevalent ${ }^{26}$ to also quantify and compare the signal-to-noise ra- ${ }^{27}$ tio (SNR) of the two transducers. The three-pulse $\mathrm{AM}^{28}$ imaging sequence was used to acquire $N=10$ frames $^{29}$ of a speckle phantom with $0.5 \mathrm{~dB} \mathrm{~cm}^{-1} \mathrm{MHz}^{-1}$ atten- ${ }^{30}$ uation. Only the full emission was used to beamform ${ }^{31}$ B-mode images of the phantom, using the same axial ${ }^{32}$ sampling and depths as the images acquired of the mi- ${ }^{33}$ croflow phantom in Section 3.2. The acquisition was re-34 peated using both the PZT transducer and the CMUT ${ }^{35}$ for the range of applied AC voltages specified in Sec-36 tion 3.2 , with a step-size of $10 \mathrm{~V}$. After beamforming, ${ }^{37}$ the power of an image line located at $x=0$ was ex- ${ }^{38}$ tracted from the images, and the SNR was quantified ${ }^{39}$ 
${ }^{1}$ as

2

${ }_{4} \quad \mathrm{SNR}=\frac{1}{N} \sum_{N=1}^{10} \frac{\sum_{m=k_{1}}^{k_{2}} \bar{y}^{2}}{\sum_{m=k_{1}}^{k_{2}}(y-\bar{y})^{2}}$,

${ }^{5}$ where $y$ is the image line and $\bar{y}$ represents the average

${ }^{6}$ of the image line across the 10 acquisitions.

7

\section{Results}

${ }^{9}$ Fig. 3 shows examples of a B-mode image and both ${ }^{10} \mathrm{a}$ two-pulse and a three-pulse AM image of the mi${ }^{11}$ croflow phantom and tissue-mimicking material, where 12the chosen ROIs are drawn on top of the images. In 13the images, it is clear that both the AM sequences ${ }^{14}$ with both probes have succeeded in removing most of ${ }^{15}$ the speckle pattern from the tissue-mimicking phan16 tom, whereas the signal from the microbubbles re${ }^{17}$ main. More speckle pattern remains in the AM images ${ }^{18}$ composed with two pulses, indicating that the three${ }^{19}$ pulse sequence outperforms the two-pulse sequence. ${ }^{20}$ The similarity of the images collected with the CMUT, ${ }^{21}$ in the left column of Fig. 3, and the PZT transducer, 22 in the right column, gives a clear indication that CEUS ${ }^{23}$ with a CMUT is possible, despite the nonlinear nature ${ }^{24}$ of the probe.

25 Equations (12) and (13) were applied to all the col${ }^{26}$ lected images, both in three-pulse AM, two-pulse AM, ${ }^{27}$ and B-mode to calculate their CTR. The mean and ${ }^{28}$ standard deviation of the CTR was calculated for each ${ }^{29}$ set of 10 frames collected with constant $V_{A C}$. Fig. 4 ${ }^{30}$ shows the resulting mean CTR values with standard ${ }^{31}$ deviations as a function of $V_{A C}$ applied to the CMUT ${ }^{32}$ for the three-pulse AM and B-mode, in addition to ${ }^{33}$ the difference between the two in decibels. Comparing ${ }^{34}$ the two modes shows that the CMUT has achieved a ${ }^{35}$ significant contrast enhancement across all the applied ${ }^{36} \mathrm{AC}$ voltages, with $30-38 \mathrm{~dB}$ higher CTR in the CEUS ${ }^{37}$ images than in the B-mode.

38 Although the theory of the three-pulse AM sequence, ${ }^{39}$ presented in Section 2, predicts that CTR should not degrade as the amount nonlinearity in the emitted sig- ${ }^{1}$ nal rises with the applied $\mathrm{AC}$ voltage, it is expected ${ }^{2}$ that the CTR varies with $\mathrm{AC}$ voltage due to the na- ${ }^{3}$ ture of the microbubble contrast agents. As the ap- ${ }^{4}$ plied voltage increases, so does the PNP of the emitted ${ }^{5}$ signal, which affects both the power and the spectral ${ }^{6}$ content of the scattered signal from the microbubbles ${ }^{7}$ [33]. Therefore, to fully understand the observed vari- ${ }^{8}$ ation in CTR seen in Fig. 4, in Fig. 5, the CTR values ${ }^{9}$ are also plotted as a function of the emitted PNP, $\mathrm{as}^{10}$ recorded by the hydrophone measurements. In addi- ${ }^{11}$ tion, the CTR of the B-mode and CEUS images col- ${ }^{12}$ lected using the PZT probe are plotted, to provide ${ }^{13}$ a reference of how the CTR varies with PNP when ${ }^{14}$ the emission from the probe is linear. The CTR for ${ }^{15}$ the PZT array is divided into two data-sets, repre- ${ }^{16}$ senting the images collected with separate syringes of ${ }^{17}$ SonoVue.

The compounded AM signals were computed from $_{20}$ the collected hydrophone recordings, using (7), and ${ }_{21}$ the annihilated power ratio was calculated as in $(14)_{22}$ Fig. 6 shows how the power ratio varies with $\mathrm{PNP}_{23}$ Had the cancellation of the signals been completely ${ }_{24}$ successful, all power would be removed and the power ${ }_{25}$ ratio would be $100 \%$. That is not the case, and it can ${ }_{26}$ be seen that the power ratio declines as PNP increases ${ }_{27}$ above approximately $455 \mathrm{kPa}$. In the region where the ${ }_{28}$ two probes' PNP overlap, the power ratios of both the ${ }_{29}$ PZT transducer and the CMUT are high, indicating ${ }_{30}$ that the emitted signals are suitable for CEUS imag- ${ }_{31}$ ing, and that the efficiency of the three-pulse sequence ${ }_{32}$ is not affected by the increase in nonlinearity in the ${ }_{33}$ emitted signal from the CMUT, as the AC voltage is ${ }_{34}$ increased.

Finally, the SNR of the image acquisition using the ${ }^{36}$ two probes at varying $\mathrm{AC}$ voltages were calculated ac- ${ }^{37}$ cording to (15). The measured SNR is given in Table $3 .^{38}$ Generally, it is seen that the SNR of the CMUT probe ${ }^{39}$ 


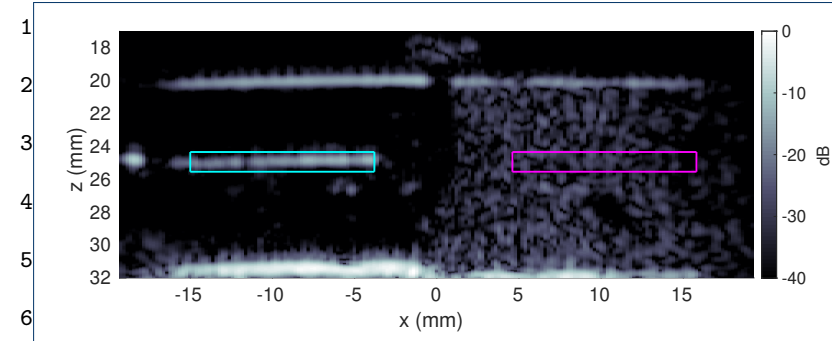

(a) CMUT: B-mode

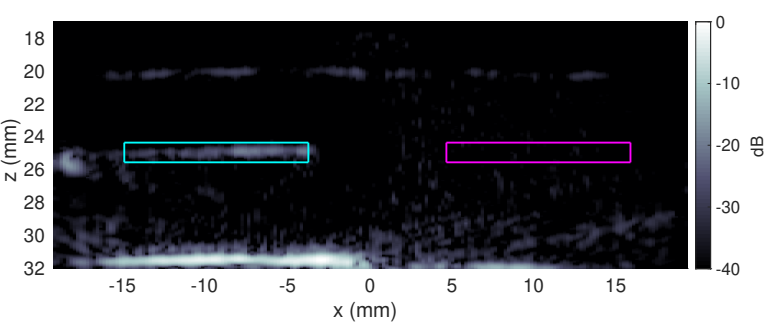

(c) CMUT: two-pulse amplitude modulation

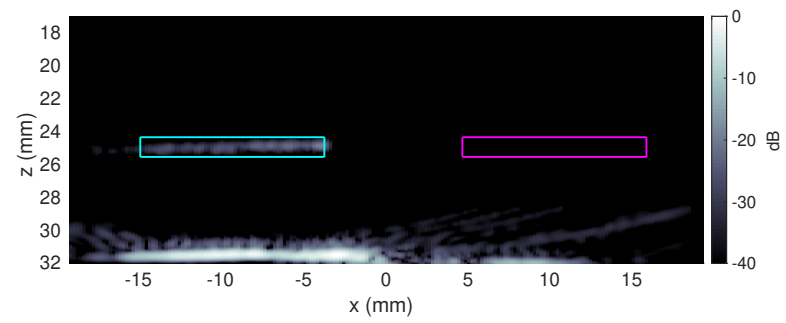

(e) CMUT: three-pulse amplitude modulation

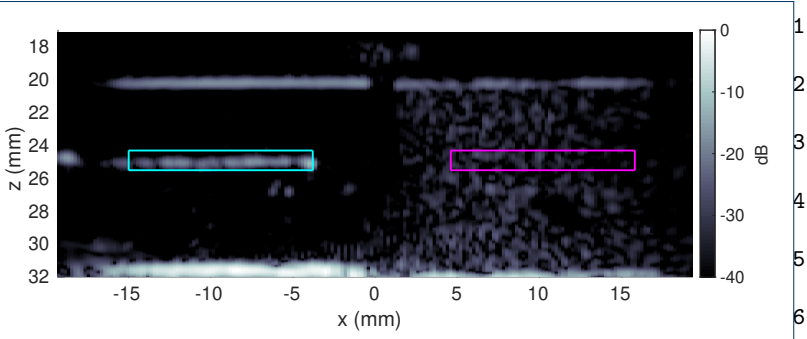

(b) PZT: B-mode

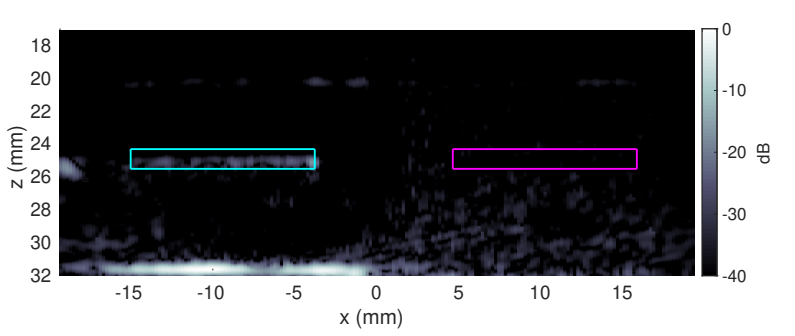

(d) PZT: two-pulse amplitude modulation

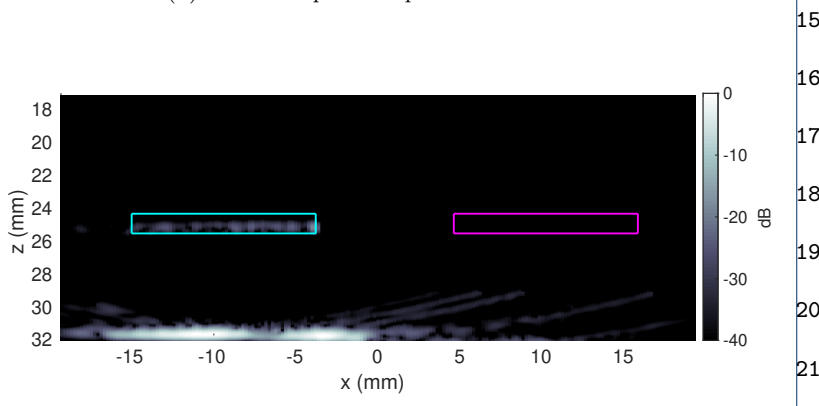

(f) PZT: three-pulse amplitude modulation

Figure 3: Example images of the experimental set-up collected with the CMUT probe (left column) and PZT ${ }^{23}$ transducer (right column). Comparing the B-mode ( $\mathrm{a}$ and b) with the two-pulse AM images (c and d) and ${ }^{24}$ the three-pulse AM image (e and f) show that the three-pulse sequence has successfully removed the speckle ${ }^{25}$ pattern inside the tissue-mimicking material (on the right of the image), while retaining the signal from the ${ }^{26}$ ${ }^{27}$ microbubbles (on the left of the image). The magenta and cyan boxes show the regions of interest used to ${ }^{27}$ 28 calculate the CTR.

29

31is lower than the PZT transducer, with a mean differ32ence of $16 \mathrm{~dB}$ across $6 \mathrm{~V}$ to $56 \mathrm{~V}$ applied AC voltage. ззFurther discussion of the data will be given in Section 345.

35

\section{Discussion}

${ }^{37} \mathrm{As}$ predicted by the theory laid out in Section 2, ${ }^{38} \mathrm{Fig}$. 4 shows that the CTR of the CEUS images col${ }^{39}$ lected with the CMUT using the three-pulse AM se- quence is not affected by the increase in nonlinear31 emission caused by an increasing $\mathrm{AC}$ voltage. Therez2 is a significant enhancement of contrast between theз3 CEUS images and the B-mode images, with an av-34 erage of $35.3 \mathrm{~dB}$ enhancement across the applied volt-35 ages. Moreover, although the CTR of the CEUS imagess6 shown in Fig. 4 declines above $46 \mathrm{~V}$, the difference be-37 tween the logarithm of the CTR of the CEUS imagess8 and the B-mode images increases from $26 \mathrm{~V}$ to $60 \mathrm{~V}, 39$ 
${ }^{1}$ Table 3: The measured SNR of the CMUT and PZT arrays across all of the applied AC voltages. The SNR is ${ }^{1}$ ${ }^{2}$ calculated according to (15) and displayed with \pm one standard deviation, $\sigma$.

\begin{tabular}{|c|c|c|c|c|c|c|c|c|c|c|c|c|}
\hline 3 & AC voltage (V) & 6 & 16 & 26 & 36 & 46 & 56 & 60 & 66 & 76 & 86 & 90 \\
\hline & CMUT SNR $\pm \sigma(\mathbf{d B})$ & $37 \pm 4$ & $50 \pm 6$ & $61 \pm 7$ & $65 \pm 6$ & $71 \pm 6$ & $72 \pm 6$ & $72 \pm 6$ & $\mathrm{~N} / \mathrm{A}$ & $\mathrm{N} / \mathrm{A}$ & $\mathrm{N} / \mathrm{A}$ & $\mathrm{N} / \mathrm{A}$ \\
\hline 5 & $\mathbf{P Z T} \mathbf{S N R} \pm \sigma(\mathbf{d B})$ & $71 \pm 5$ & $64 \pm 4$ & $75 \pm 5$ & $80 \pm 6$ & $81 \pm 7$ & $84 \pm 5$ & $\mathrm{~N} / \mathrm{A}$ & $87 \pm 5$ & $92 \pm 5$ & $92 \pm 5$ & $85 \pm 5$ \\
\hline
\end{tabular}

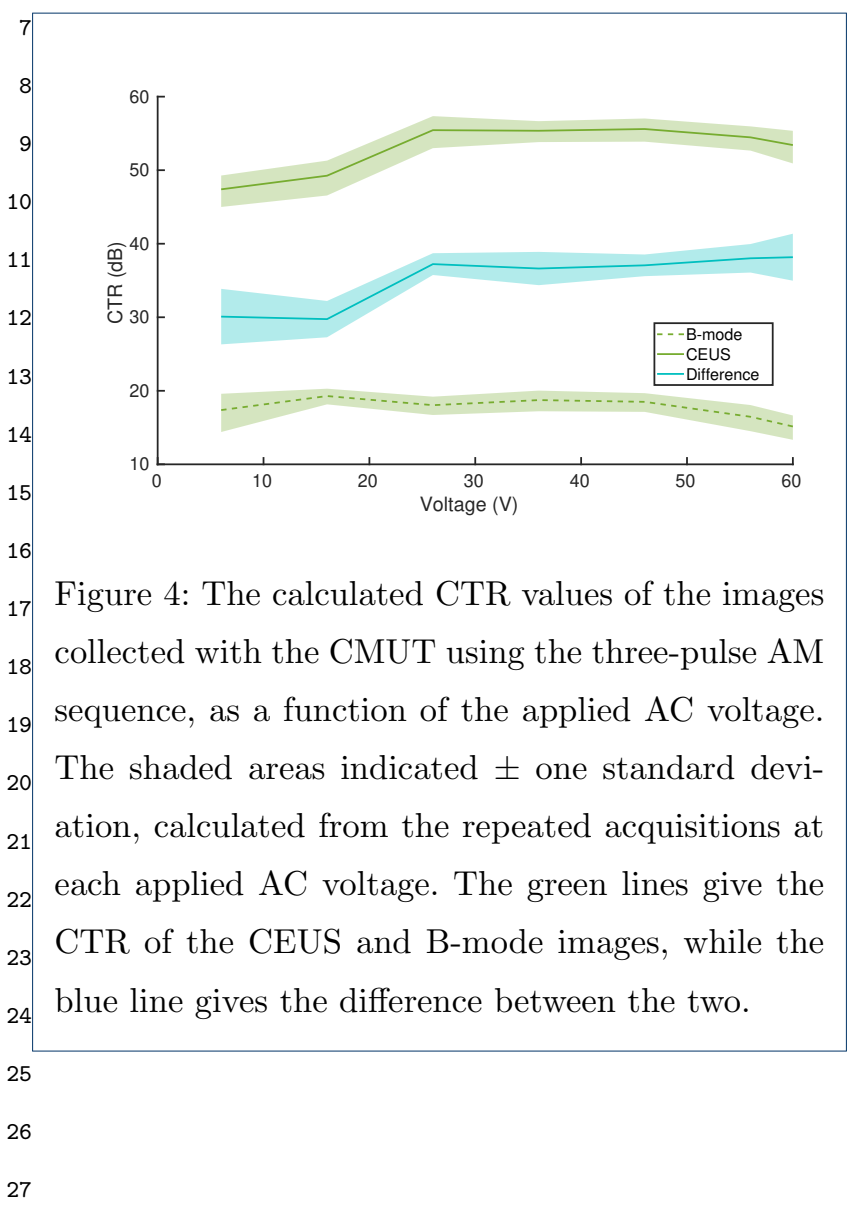

${ }^{28}$ demonstrating that the enhancement of the contrast ${ }^{29}$ caused by the three-pulse AM sequence is not affected ${ }^{30}$ by the applied AC voltage. On the other hand, it can ${ }^{31}$ bee seen in Fig. 5 that the two-pulse AM sequence has ${ }^{32}$ not accomplished the same enhancement. Clearly, re${ }^{33}$ ducing the number of emissions in the minor sequence, ${ }^{34}$ would benefit the frame rate. However, the average dif${ }^{35}$ ference in CTR between the CEUS images and the B${ }^{36}$ mode images for the two-pulse AM sequence is only ${ }^{37} 9.1 \mathrm{~dB}$. Thus, CEUS imaging using a CMUT should ${ }^{38}$ use the three-pulse AM sequence, despite the decrease ${ }^{39}$ in frame rate this will lead to.

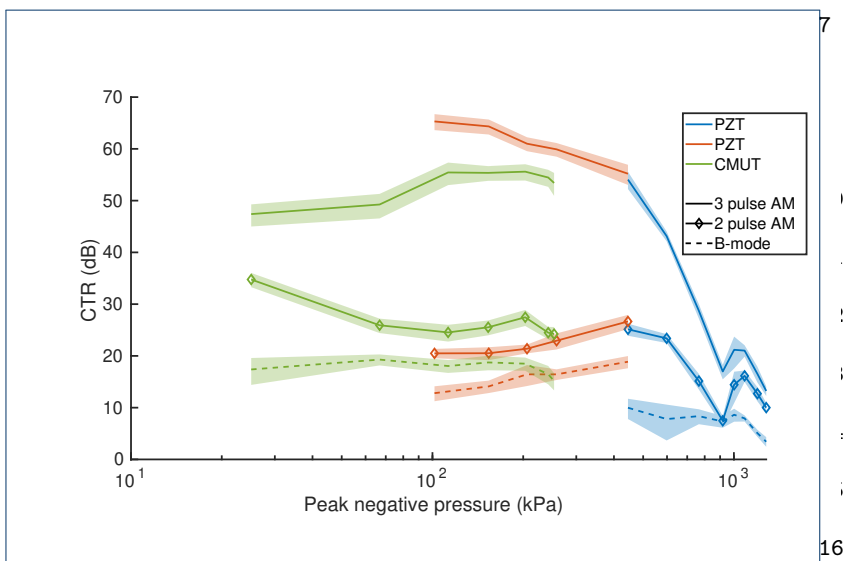

Figure 5: The calculated CTR values of the images ${ }_{17}^{16}$ collected with the CMUT and PZT transducer, as a 18 function of the emitted peak-negative-pressure. The 19 CTR of three-pulse AM images are plotted with 20 solid lines, of the two-pulse AM images with solid 21 lines with diamonds, and the CTR of the B-mode 22 images are plotted with dotted lines. The shaded ar- 23 eas indicated \pm one standard deviation, calculated ${ }_{24}$ from the repeated acquisitions at each applied AC 25 voltage.

26

\section{8}

The CTR of the CEUS images follows the expected ${ }^{29}$ trend; it increases initially, before starting to de- ${ }^{30}$ crease at higher voltages. This progression is caused ${ }^{31}$ by the back-scattering properties of microbubbles. $\mathrm{At}^{32}$ low pressures, the nonlinear component of the back- ${ }^{33}$ scattering from microbubbles is proportional to the in- ${ }^{34}$ cident acoustic pressure [33, 34], and Emmer et al. [35] $]^{35}$ showed that SonoVue exhibits a low pressure thresh- ${ }^{36}$ old, under which the bubbles do not scatter nonlin- ${ }^{37}$ early. On the other hand, at high pressures, the $\mathrm{CTR}^{38}$ is expected to drop because the microbubbles start ${ }^{39}$ 


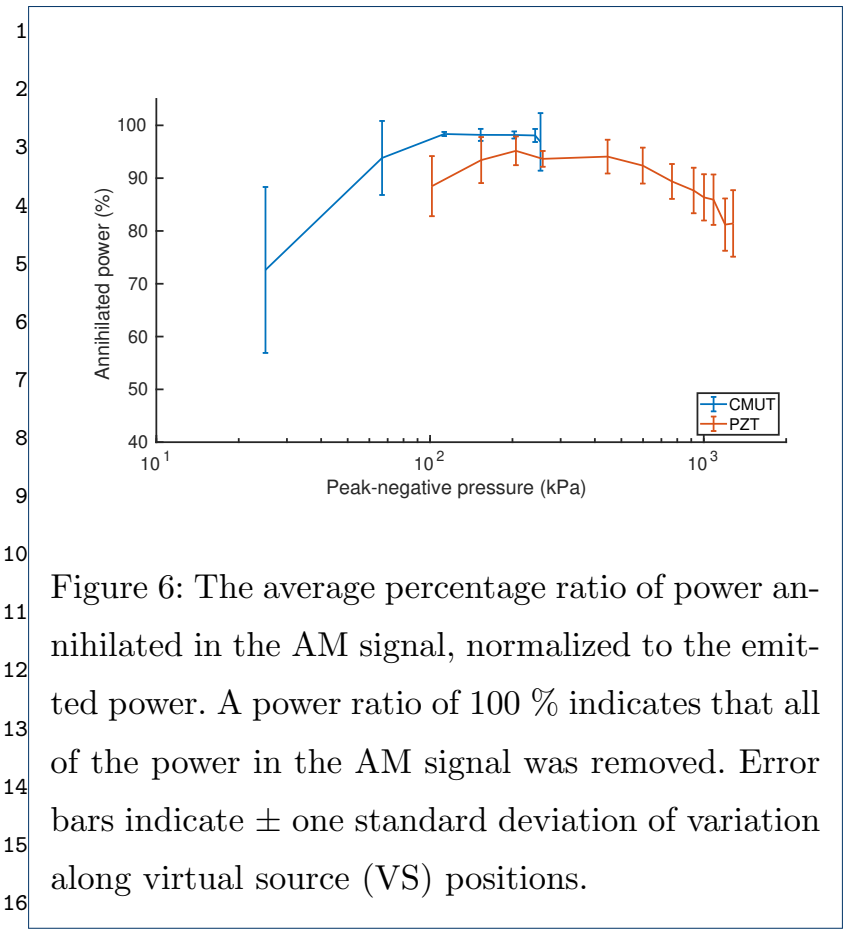

17

18

${ }_{19}$ to degrade, through processes such as rupture of the 20lipid shell, diffusion of the gas core, or fragmentation ${ }_{21}[36-38]$. A decline in CTR of the CEUS images col${ }_{22}$ lected with the CMUT is observed in Fig. 4 starting ${ }_{23}$ at $46 \mathrm{~V}$, and correspondingly in Fig. 5 at $203 \mathrm{kPa}$. How${ }_{24} \mathrm{ever}$, this decline in CTR does not point to a failure ${ }_{25}$ of the three-pulse AM sequence, but rather a natu${ }_{26} \mathrm{ral}$ consequence of the properties of the microbubbels. ${ }_{27}$ According to de Jong et al. [33], SonoVue can with${ }_{28}$ stand up to $150 \mathrm{kPa}$ before it starts to deform, and it ${ }_{29}$ is destroyed with a few repeated ultrasound exposures 30 above approximately $300 \mathrm{kPa}$. This corresponds to the ${ }_{31}$ range at which the CTR in Fig. 5 starts to decline, tak${ }_{32}$ ing into account the attenuation the emitted signal will ${ }_{33}$ experience before reaching the microbubbles inside the ${ }_{34}$ phantom. Thus, it has been shown that the ideal pres${ }_{35}$ Sure range to perform CEUS imaging with a CMUT ${ }_{36}$ lies approximately between $100 \mathrm{kPa}$ to $200 \mathrm{kPa}$.

37 Moreover, the same downwards trend in CTR of ${ }^{38}$ the CEUS images above approximately $100 \mathrm{kPa}$ is ob${ }^{39}$ served for the images collected using the PZT trans- ducer as for the images collected using the CMUT, ${ }^{1}$ further reinforcing that the decline is not caused $b^{2}$ a deterioration of the three-pulse AM sequence. The ${ }^{3}$ PZT transducer displays a rapid decline in CTR above ${ }^{4}$ $455 \mathrm{kPa}$, likely caused by both the microbubble disrup- ${ }^{5}$ tion discussed above, but also by nonlinear propaga- ${ }^{6}$ tion in the image medium. The ratio of annihilated ${ }^{7}$ power shown in Fig. 6 also declines above $455 \mathrm{kPa},{ }^{8}$ pointing to a discrepancy in the emitted pulses. Obser- ${ }^{9}$ vation of the recorded hydrophone signals reveal saw- ${ }^{10}$ tooth like pulse shapes, indicative of nonlinear propa- ${ }^{11}$ gation [39]. This distortion of the emitted pulses will ${ }^{12}$ differ between the full amplitude and half amplitude ${ }^{13}$ emissions, and thus cause the cancellation in (7) to ${ }^{14}$ fail.

Although the same AC voltage was applied to the ${ }^{18}$ CMUT and the PZT transducer, up to the maximum ${ }^{19}$ tolerable AC voltage of the CMUT, the PZT trans- ${ }^{20}$ ducer emits higher PNP than the CMUT. The two ${ }^{21}$ probes have some overlap in their area of operation be- ${ }^{22}$ tween $101 \mathrm{kPa}$ to $258 \mathrm{kPa}$. In this region, both probes $^{23}$ exhibit a significant contrast enhancement, with an av- ${ }^{24}$ erage difference in CTR between the CEUS images and ${ }^{25}$ the B-mode images of $49.9 \mathrm{~dB}$ and $37.4 \mathrm{~dB}$ for the $\mathrm{PZT}^{26}$ transducer and CMUT, respectively. The fact that the ${ }^{27}$ PZT transducer overall performs better contrast en- ${ }^{28}$ hancement than the CMUT is likely caused by the ${ }^{29}$ PZT transducer's superior SNR. At a constant emit- ${ }^{30}$ ted pressure, the back-scattering from the microbub- ${ }^{31}$ bles will generally be the same for the PZT transducer ${ }^{32}$ and CMUT. Yet, the CTR can differ, if the amount of ${ }^{33}$ received noise is higher for the CMUT. As shown in Ta- ${ }^{34}$ ble 3 the CMUT has a lower SNR than the PZT trans- ${ }^{35}$ ducer at all applied voltages. This is expected, since ${ }^{36}$ the probe is built in-house, whereas the PZT trans- ${ }^{37}$ ducer is a commercial probe and has superior shielding ${ }^{38}$ from electromagnetic interference noise. 39 
1 Yet, the quantification of the annihilated power ${ }^{2}$ shown in Fig. 6 shows that the CMUT generally out${ }^{3}$ performs the PZT transducer. The power annihilation ${ }^{4}$ ratio quantifies how much power that remains in the ${ }^{5}$ compounded emission and thus gives an indication of ${ }^{6}$ how well the transducers are able to perform contrast ${ }^{7}$ enhancement imaging. The cancellation of the emit${ }^{8}$ ted pulses is more effective for the CMUT than the ${ }^{9} \mathrm{PZT}$ transducer, indicating that array effects in the ${ }^{10} \mathrm{CMUT}$ is not causing significant degradation of the ${ }^{11}$ contrast enhancement. In the region where the two ${ }^{12}$ probes' PNPs overlap, the compounding of the AM ${ }^{13}$ signal from the CMUT leaves an average of $2 \%$ of the ${ }^{14}$ emitted power, while for the PZT transducer the av${ }^{15}$ erage remaining power is $7 \%$. When using the emis${ }^{16}$ sions for imaging, the amount of remaining power will ${ }^{17}$ transfer to the scattered signals and impair the ability ${ }^{18}$ to cancel linear signal from the tissue, thus lowering ${ }^{19}$ the CTR. Moreover, both probes exhibit an increase ${ }^{20}$ in the annihilated power with applied AC voltage, as ${ }^{21}$ well as an increased standard deviation of the annihi${ }^{22}$ lated power at low applied AC voltages. This further ${ }^{23}$ supports that the nonlinear emission from the CMUT ${ }^{24}$ does not negatively impact the creation of CEUS im${ }^{25}$ ages using the proposed three-pulse AM sequence.

26

\section{${ }^{27} 6$ Conclusion}

${ }^{28}$ Investigation of the creation of CEUS images with a ${ }^{29} \mathrm{CMUT}$ has shown that the probe's inherent nonlin${ }^{30}$ earity does not affect its performance, and that high ${ }^{31}$ contrast CEUS images can be created using a three${ }^{32}$ pulse AM sequence. The fact that the performance of ${ }^{33}$ the CMUT probe is not dependent on the harmon${ }^{34}$ ics of its emissions has been demonstrated theoreti${ }^{35}$ cally, using quantification of CTR of CEUS images of ${ }^{36}$ microbubbles, and using hydrophone measurements of ${ }^{37}$ the emitted signals. Theoretically, it has been shown ${ }^{38}$ that there is no reason why the emitted harmonics will ${ }^{39}$ affect the CEUS images. This was confirmed by the ac- quired CEUS images, where an average of $37.4 \mathrm{~dB}_{\text {con- }}{ }^{1}$ trast enhancement was achieved, using the three-pulse ${ }^{2}$ AM sequence. Comparison of the CTR of the images ${ }^{3}$ obtained using the proposed three-pulse AM sequence ${ }^{4}$ with the CTR of images obtained using only 2 emis- $^{5}$ sions showed a significant advantage of the three-pulse ${ }^{6}$ $\mathrm{AM}$, as the two-pulse AM sequence gave only an aver- ${ }^{7}$ age of $9.07 \mathrm{~dB}$ contrast enhancement. Thus, a higher ${ }^{8}$ number of emissions in the minor sequence must be ${ }^{9}$ used, despite its negative impact on the frame rate. ${ }^{10}$ Further, comparison of the performance of the $\mathrm{CMUT}^{11}$ with a PZT transducer of similar design showed a com- ${ }^{12}$ parable amount of contrast enhancement, as the im- ${ }^{13}$ ages produced with the PZT transducer achieved $\mathrm{an}^{14}$ average of $49.9 \mathrm{~dB}$ contrast enhancement. The $\mathrm{PZT}^{15}$ transducer achieved higher CTR, but also higher SNR, ${ }^{16}$ and improvement of the design of the CMUT, espe- ${ }^{17}$ cially focused on noise shielding, will likely improve ${ }^{18}$ its contrast enhancement abilities. Finally, hydrophone ${ }^{19}$ recordings and quantification of the annihilated power ${ }^{20}$ in the minor sequence showed that in the region of ${ }^{21}$ overlapping PNPs, the CMUT annihilated $98 \%$ of the ${ }^{22}$ power, whereas the PZT transducer only annihilated ${ }^{23}$ $93 \%$. Thus, more power was cancelled in the CMUT's ${ }^{24}$ minor sequence than in the PZT transducer's, fur- ${ }^{25}$ ther reinforcing that the difference between the $\mathrm{CTR}^{26}$ produced by the CMUT and the PZT transducer is ${ }^{27}$ caused by the probes respective receive capabilities, ${ }^{28}$ rather than the emitted harmonics. Thus, future use ${ }^{29}$ of CMUT technology for CEUS images show great po- ${ }^{30}$ tential, and it will be possible to take advantage of the ${ }^{31}$ wide bandwidth, low emission pressure, and favorable ${ }^{32}$ sensitivity features of CMUTs for CEUS imaging. $\quad 33$

$\begin{array}{ll}7.1 \text { Abbreviations } & 36\end{array}$ $\begin{array}{ll}\text { AM Amplitude modulation. } & 37\end{array}$ CEUS Contrast enhanced ultrasound. 38 CMUT Capacitive micromachined ultrasonic transducer. CTR Contrast-to-tissue ratio. 
${ }^{1}$ PI Pulse inversion.

2PNP Peak-negative-pressure.

${ }_{3} \mathrm{PZT}$ Lead zirconate titanate.

ROI Region-of-interest.

${ }^{4}$ SNR Signal-to-noise ratio.

5SRI Super-resolution ultrasound imaging.

${ }_{6}$ SVD singular value decomposition.

VS Virtual source.

87.2 Ethics approval and consent to participate

Not applicable.

107.3 Consent for publication

${ }_{11}$ Not applicable.

${ }^{12} 7.4$ Availability of data and material

${ }_{13}$ The datasets used and/or analysed during the current study are available 14

from the corresponding author on reasonable request.

157.5 Competing interests

${ }_{16}$ The authors declare that they have no competing interests.

${ }^{17} 7.6$ Funding

18 This work was financially supported by grant 82-2014-4 from the Danish National Advanced Technology Foundation, by grant 7050-00004B from Innovation Fund Denmark, and by BK Medical, Herlev, Denmark. 20

217.7 Authors' contributions

S.H. Øygard wrote the main manuscript text and prepared the figures. M.

${ }^{22} \mathrm{~L}$. Ommen manufactured the phantom and designed part of the

23 experimental set-up. B. G. Tomov assisted with technical measurements. S. ${ }_{24}$ E. Diederichsen manufactured the CMUT probe. E. V. Thomsen, N. B.

Larsen, M. B. Stuart, and J. A. Jensen contributed to designing the study

25 and discussed the results. All authors reviewed the manuscript.

26

$27^{7.8}$ Acknowledgments

Not applicable.

28

8 Appendix

${ }^{29}$ The tree emissions given in (3) - (5) can be written on the compact form 30 seen in (2) as

31

32

$$
p_{A}(t)=p_{L}+\sum_{h=2}^{\infty} p_{H}
$$$$
p_{B}(t)=0.5 p_{L}+0.5 \sum_{h=2}^{\infty} p_{H},
$$

34

$$
p_{C}(t)=0.5 p_{L}+0.5 \sum_{h=2}^{\infty} p_{H} .
$$

${ }^{36}$ The emissions in this form are inserted into (9);

37

38

$$
\begin{aligned}
y_{A M}(t)= & a_{1} p_{A}+a_{2} p_{A}{ }^{2}+a_{3} p_{A}{ }^{3} \\
& -a_{1} p_{B}-a_{2} p_{B}{ }^{2}-a_{3} p_{B}{ }^{3} \\
& -a_{1} p_{C}-a_{2} p_{C}{ }^{2}-a_{3} p_{B}{ }^{3}
\end{aligned}
$$$$
39
$$

$$
\begin{aligned}
= & a_{1}\left(p_{L}+\sum_{h=2}^{\infty} p_{H}\right)+a_{2}\left(p_{L}+\sum_{h=2}^{\infty} p_{H}\right)^{2} \\
& +a_{3}\left(p_{L}+\sum_{h=2}^{\infty} p_{H}\right)^{3}-a_{1}\left(0.5 p_{L}+0.5 \sum_{h=2}^{\infty} p_{H}\right)^{2} 3 \\
& -a_{2}\left(0.5 p_{L}+0.5 \sum_{h=2}^{\infty} p_{H}\right)^{2}-a_{3}\left(p_{L}+\sum_{h=2}^{\infty} p_{H}\right)^{3} 4 \\
& -a_{1}\left(0.5 p_{L}+0.5 \sum_{h=2}^{\infty} p_{H}\right) \\
& -a_{2}\left(0.5 p_{L}+0.5 \sum_{h=2}^{\infty} p_{H}\right)^{2}-a_{3}\left(p_{L}+\sum_{h=2}^{\infty} p_{H}\right)^{3} \cdot
\end{aligned}
$$

Canceling terms and expanding the parenthesis gives

$$
\begin{aligned}
y_{A M}(t)= & 0.5 a_{2} p_{L}^{2}+0.5 a_{2}\left(\sum_{h=2}^{\infty} p_{H}\right)^{2} \\
& +a_{2} p_{L}\left(\sum_{h=2}^{\infty} p_{H}\right)+2.25 a_{3} p_{L}\left(\sum_{h=2}^{\infty} p_{H}\right)^{2} \\
& +2.25 a_{3} p_{L}^{2}\left(\sum_{h=2}^{\infty} p_{H}\right) \\
& +0.75 a_{3}\left(\sum_{h=2}^{\infty} p_{H}\right)^{3}+0.75 a_{3} p_{L}{ }^{3} .
\end{aligned}
$$

.

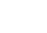

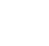


${ }^{1}$ Author details

$2^{1}$ Center for Fast Ultrasound Imaging, Department of Health Technology,

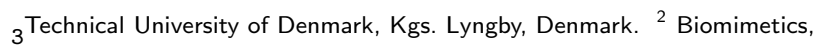
Department of Health Technology, Technical University of Denmark, Kgs. ${ }^{4}$ Lyngby, Denmark. ${ }^{3}$ MEMS - Applied Sensors, Department of Health 5Technology, Technical University of Denmark, Kgs. Lyngby, Denmark.

\section{${ }^{6}$ References}

7 1. Oralkan, O., Ergun, A.S., Johnson, J.A., Karaman, M., Demirci, U., Kaviani, K., Lee, T.H., Khuri-Yakub, B.T.: Capacitive micromachined ultrasonic transducers: Next-generation arrays for acoustic imaging?

9 IEEE Trans. Ultrason., Ferroelec., Freq. Contr. 49, 1596-1610 (2002)

10 2. Haller, M.I., Khuri-Yakub, B.T.: A surface micromachined electrostatic ultrasonic air transducer. In: Proc. IEEE Ultrason. Symp., vol. 2, pp. 1241-1244 (1994)

12 3. Dietrich, C.F., Averkiou, M., Nielsen, M.B., Barr, R.G., Burns, P.N.,

13 Calliada, F., Cantisani, V., Choi, B., Chammas, M.C., Clevert, D., et al.: How to perform contrast-enhanced ultrasound (CEUS). Ultrasound international open 4(1), 2 (2018)

15 4. Tang, M.X., Mulvana, H., Gauthier, T., Lim, A.K.P., Cosgrove, D.O.,

16 Eckersley, R.J., Stride, E.: Quantitative contrast-enhanced ultrasound 17 imaging: a review of sources of variability. Interface Focus $1(4)$, 520-539 (2011)

18 5. Errico, C., Pierre, J., Pezet, S., Desailly, Y., Lenkei, Z., Couture, O.,

19 Tanter, M.: Ultrafast ultrasound localization microscopy for deep super-resolution vascular imaging. Nature 527, 499-502 (2015)

6. Christensen-Jeffries, K., Browning, R.J., Tang, M., Dunsby, C.,

21 Eckersley, R.J.: In vivo acoustic super-resolution and super-resolved

22 velocity mapping using microbubbles. IEEE Trans. Med. Imag. 34(2), 433-440 (2015)

7. Novell, A., Bouakaz, A., Legros, M., Felix, N.: Nonlinear contrast

24 imaging with capacitive micromachined transducers. Proc. IEEE

25 Ultrason. Symp. (2008)

8. Leighton, T.G.: The Acoustic Bubble. Elsevier Inc., London (1994)

9. Martin, K.H., Lindsey, B.D., Ma, J., Lee, M., Li, S., Foster, F.S.,

27 Jiang, X., Dayton, P.A.: Dual-frequency piezoelectric transducers for

28 contrast enhanced ultrasound imaging. Sensors, 20825-20842 (2014)

29 10. Kamaya, A., Machtaler, S., Sanjani, S.S., Nikoozadeh, A., Sommer, F.G., Khuri-Yakub, B.T., Willmann, J.K., Desser, T.S.: New

30 technologies in clinical ultrasound. Seminars in Roentgenology,

$31 \quad 214-223(2013)$

32 11. Eckersley, R.J., Chin, C.T., Burns, P.N.: Optimising phase and amplitude modulation schemes for imaging microbubble contrast

33 agents at low acoustic power. Ultrasound Med. Biol. 31(2), 213-219 34 (2005)

35 12. Savoia, A.S., Caliano, G., Pappalardo, M.: A CMUT probe for medical ultrasonography: From microfabrication to system integration. IEEE

36 Trans. Ultrason., Ferroelec., Freq. Contr. 59(6), 1127-1138 (2012)

3713. Lohfink, A., Eccardt, P.C.: Investigation of nonlinear CMUT behavior.

38 In: Proc. IEEE Ultrason. Symp., pp. 585-588 (2005)

14. Miller, D.L.: Ultrasonic detection of resonant cavitation bubbles in a

39 flow tube by their second-harmonic emissions. Ultrasonics 19, 217-224
(1981)

1

15. Simpson, D.H., Chin, C.T., Burns, P.N.: Pulse inversion Doppler: A 2 new method for detecting nonlinear echoes from microbubble contrast 3 agents. IEEE Trans. Ultrason., Ferroelec., Freq. Contr. 46(2), 372-382 (1999)

16. Brock-Fischer, G.A., Poland, M.D., Rafter, P.G.: Means for increasing 5 sensitivity in non linear ultrasound systems. US Patent (5577505) 6 (1996)

17. Oygard, S.H., Ommen, M.L., Engholm, M., Schou, M., Diederichsen, ${ }^{7}$ S.E., Thomsen, E.V., Stuart, M.B., Jensen, J.A.: Investigating a 8 CMUT's ability to achieve non-linear contrast enhancement. In: Proc. 9 IEEE Ultrason. Symp., pp. 1-4 (2020)

18. Fouan, D., Bouakaz, A.: Investigation of classical pulse sequences for 10 contrast-enhanced ultrasound imaging with a CMUT probe. IEEE 11 Trans. Ultrason., Ferroelec., Freq. Contr. 63(10), 1496-1503 (2016) 12

19. Zhou, S., Reynolds, P., Hossack, J.: Pre-compensated excitation waveform to suppress second harmonic generation in MEMS 13 electrostatic transducers. In: Proc. IEEE Ultrason. Symp., pp. 477-48014 (2003)

20. Lei, A., E.Diederichsen, S., Hansen, S.M., Stuart, M.B., Bagge, J.P., 15 Jensen, J.A., Thomsen, E.V.: Elimination of second-harmonics in 16 CMUTs using square pulse excitation. In: Proc. IEEE Ultrason. Symp., 17 pp. 1-4 (2016)

21. Bouakaz, A., Frigstad, S., Cate, F.T., de Jong, N.: Super harmonic imaging: a new imaging technique for improved contrast detection. 19 Ultrasound Med. Biol. 28(1), 59-68 (2002) 20

22. Certon, D., Teston, F., Patat, F.: A finite difference model for CMUT 21 devices. IEEE Trans. Ultrason., Ferroelec., Freq. Contr. 52(12), 2199-2210 (2005)

23. Kinsler, L.E., Frey, A.R., Coppens, A.B., Sanders, J.V.: Fundamentals 23 of Acoustics, 3rd edn. John Wiley \& Sons, New York (1982)

24. Lohfink, A., Eccardt, P.-C.: Linear and nonlinear equivalent circuit modeling of CMUTs. IEEE Trans. Ultrason., Ferroelec., Freq. Contr. 25 52(12), 2163-2172 (2005) 26

25. Meynier, C., Teston, F., Certon, D.: A multiscale model for array of 27 capacitive micromachined ultrasonic transducers. J. Acoust. Soc. Am. 128(5), 2549-2561 (2010)

26. Novell, A., Bouakaz, A., Legros, M., Felix, N.: Exploitation of 29 capacitive micromachined transducers for nonlinear ultrasound 30 imaging. Proc. IEEE Ultrason. Symp., 2733-2743 (2009)

27. Ommen, M.L., Schou, M., Zhang, R., Hoyos, C.A.V., Jensen, J.A., 31 Larsen, N.B., Thomsen, E.V.: 3D printed flow phantoms with fiducial 32 markers for super-resolution ultrasound imaging. In: Proc. IEEE 33 Ultrason. Symp., pp. 1-4 (2018)

28. Lai, S.S.M., Yiu, B.Y.S., Poon, A.K.K., Yu, A.C.H.: Design of 34 anthropomorphic flow phantoms based on rapid prototyping of 35 compliant vessel geometries. Ultrasound Med. Biol. 39(9), 1654-1664 36 (2013)

29. Olesen, J.B., Villagómez Hoyos, C.A., Møller, N.D., Ewertsen, C., 37 Hansen, K.L., Nielsen, M.B., Bech, B., Lönn, L., Traberg, M.S., 38 Jensen, J.A.: Non-invasive estimation of pressure changes using 2-D 39 

vector velocity ultrasound: An experimental study with in-vivo

2 examples. IEEE Trans. Ultrason., Ferroelec., Freq. Contr. 65(5),

30. Diederichsen, S.E.: Micromachined integrated transducers for

$4 \quad$ ultrasound imaging. PhD thesis, Denmark Technical University (2020)

531. Jensen, J.A., Nikolov, S., Gammelmark, K.L., Pedersen, M.H.:

Synthetic aperture ultrasound imaging. Ultrasonics 44, 5-15 (2006)

32. Oygard, S.H., Ommen, M.L., Engholm, M., Schou, M., Diederichsen,

7 S.E., Thomsen, E.V., Stuart, M.B., Jensen, J.A.: Investigating a

8 CMUT's ability to achieve non-linear contrast enhancement. In: Proc. IEEE Ultrason. Symp., pp. 1-4 (2020) microbubble vibration. Ultrasound Med. Biol. 33, 941-949 (2007)

${ }^{16}$ 36. Dayton, P.A., Morgan, K.E., Kilbanov, A.L., Brandenburger, G.H.,

17 Ferrara, K.W.: Optical and acoustical observations of the effects of 


\section{Supplementary Files}

This is a list of supplementary files associated with this preprint. Click to download.

- LaTexFiles.zip 